\title{
Approaching the sensitized lung patient: risk assessment for donor acceptance
}

\author{
Massimo Mangiola ${ }^{1}$, Marilyn Marrari ${ }^{2}$, Qingyong $\mathrm{Xu}^{2}$, Pablo G. Sanchez ${ }^{3}$, Adriana Zeevi ${ }^{2}$ \\ ${ }^{1}$ NYU Langone Transplant Institute, New York, NY, USA; ${ }^{2}$ Department of Pathology, University of Pittsburgh Medical Center, Pittsburgh, PA, \\ USA; ${ }^{3}$ Department of Cardiothoracic Surgery, University of Pittsburgh Medical Center, Pittsburgh, PA, USA \\ Contributions: (I) Conception and design: M Mangiola, A Zeevi; (II) Administrative support: None; (III) Provision of study materials or patients: M \\ Mangiola, A Zeevi, PG Sanchez; (IV) Collection and assembly of data: M Mangiola, A Zeevi; (V) Data analysis and interpretation: M Mangiola, M \\ Marrari, A Zeevi; (VI) Manuscript writing: All authors; (VII) Final approval of manuscript: All authors. \\ Correspondence to: Adriana Zeevi, PhD. Department of Pathology, University of Pittsburgh Medical Center, Pittsburgh, PA, USA. \\ Email: zeevia@upmc.edu.
}

\begin{abstract}
The presence of HLA antibodies is widely recognized as a barrier to solid organ transplantation, and for lung transplant candidates, it has a significant negative impact on both waiting time and waiting list mortality. Although HLA antibodies have been associated with a broad spectrum of allograft damage, precise characterization of these antibodies in allosensitized candidates may enhance their accessibility to transplant. The introduction of Luminex-based single antigen bead (SAB) assays has significantly improved antibody detection sensitivity and specificity, but SAB alone is not sufficient for risk-stratification. Functional characterization of donor-specific antibodies (DSA) is paramount to increase donor accessibility for allosensitized lung candidates. We describe here our approach to evaluate sensitized lung transplant candidates. By employing state-of-the-art technologies to assess histocompatibility and determine physiological properties of circulating HLA antibodies, we can provide our Clinical Team a better risk assessment for lung transplant candidates and facilitate a "road map" to transplant. The cases presented in this paper illustrate the "individualized steps" taken to determine calculated panel reactive antibodies (cPRA), titer and complement-fixing properties of each HLA antibody present in circulation. When a donor is considered, we can better predict the risk associated with potentially crossing HLA antibodies, thereby allowing the Clinical Team to approach allosensitized lung patients with an individualized medicine approach. To facilitate safe access of sensitized lung transplant candidates to potential donors, a synergy between the histocompatibility laboratory and the Clinical Team is essential. Ultimately, donor acceptance is a decision based on several parameters, leading to a risk-stratification unique for each patient.
\end{abstract}

Keywords: HLA antibodies; donor-specific antibodies (DSA); allosensitization; lung transplant; risk stratification; C1q; titer

Submitted Nov 27, 2020. Accepted for publication May 14, 2021.

doi: $10.21037 /$ jtd-2021-21

View this article at: https://dx.doi.org/10.21037/jtd-2021-21

\section{Introduction}

HLA allosensitization is widely recognized as a barrier to solid organ transplantation. For lung transplant candidates, it has a significant negative impact on both waiting time and waiting list mortality $(1,2)$. The number of lung transplant candidates listed with unacceptable HLA antigens in the United Network Organ Sharing (UNOS) database increased from $8 \%$ in 2006 to $22 \%$ in 2016 (2). Over the same period of time, in a single center large cohort, $35 \%$ of candidates were considered allosensitized (1). In both of these studies the HLA calculated panel-reactive antibody (cPRA) was used as a continuous measure for the level of allosensitization $(1,2)$. The overall range of allosensitization 
reported in the literature (12-42\%) is directly dependent on the center-specific algorithm to determine unacceptable antigens, which is based on different testing cutoff values and on other HLA antibody characteristics, such as complement binding (3-5). Many lung transplant programs will avoid any donor-specific antibodies (DSA) at the time of organ allocation, thus significantly limiting sensitized patients' access to transplantation (6-8). Highly sensitized patients with cPRA $>50 \%$ and $>75 \%$ have been shown to have a significantly smaller donor pool, resulting in longer waiting times, and an increased risk of death (1). Similar to patients awaiting a lung transplant, allosensitization in pediatric and adult heart transplant candidates has been shown to negatively correlate with the likelihood of transplantation and positively correlate with an increased risk of waiting list mortality (9-11).

Several studies have reported adverse outcomes after lung transplant in the presence of any panelreactive antibodies $(12,13)$, while others showed that avoiding DSA in sensitized recipients was associated with outcomes comparable to non-sensitized patients (14). Transplantation in the presence of pre-formed DSA has been associated with the development of bronchiolitis obliterative syndrome (BOS) (6) or increased mortality at 1 year post-transplant (15).

One approach to increase access to transplant for broadly sensitized solid organ candidates is to attempt antibody removal using various desensitization protocols shown to be successful in renal recipients and to a lesser degree in thoracic candidates (16). The experience with desensitization in lung transplantation has been limited to small cohorts without clear benefits reported $(7,8)$. In a large cohort study, the Toronto Lung transplant group reported on a perioperative regimen for patients with cPRA $>30 \%$ and positive DSA to achieve equivalent outcomes as compared to nonsensitized patients. However, there was insufficient information on the level and characteristics of pre-formed DSA in this cohort to evaluate the impact of the perioperative regimen to diminish or remove the HLA antibody (17).

Although HLA antibodies have been shown to be associated with a broad spectrum of allograft damage, not all HLA antibodies are equal (18-20). Luminexbased single antigen bead (SAB) assays have improved not only detection sensitivity and specificity but also the functional characterization of DSA. Additionally, the introduction of molecular HLA genotyping allowed for better discrimination of donor antigens and has improved our ability to risk-stratify candidates prior to transplant. These advances in HLA antibody testing, including interpretation and limitations of various techniques, have been summarized in several consensus publications $(21,22)$. Characteristics that have been associated with pathogenesis and deleterious effects of DSA in solid organ transplants include HLA-DQ specificity $(3,23,24)$, high titer $(25,26)$, and the ability to bind complement (27-32).

In this paper, we summarize our approach to evaluate sensitized lung transplant candidates using the state-of-theart technologies available to assess histocompatibility and physiological properties of circulating HLA antibodies. We believe that incorporating all the characteristics of HLA antibodies, including allele specificity, titer and complement binding capability, can provide clinicians a better "road map" to assess who may be transplanted without intervention or who may benefit from a pre-transplant desensitization approach. Furthermore, the risk assessment should also take into consideration potential memory responses based on the history of antibody testing and other recipient characteristics. The cases discussed below will illustrate our overall strategy.

\section{Materials and methods}

\section{Luminex single antigen beads IgG testing}

Sera were tested for the presence of IgG anti-HLA antibodies on the Luminex platform using a solid-phase SAB assay (LabScreen Single Antigen - One Lambda Thermo Fisher, West Hills, CA), In this assay, each bead is coated with a unique HLA allele representative of the most common Class I and Class II HLA alleles in the population. If present in the patient serum, HLA antibodies will bind to specific HLA antigen-coated beads and are detected by the addition of a phycoerythrin-conjugated secondary anti$\mathrm{IgG}$ antibody. Beads with normalized mean fluorescence intensity (MFI) $\geq 1,000$ were considered positive. cPRA, which is based on the frequency of the unacceptable antigens in the United Network for Organ Sharing (UNOS) deceased donor pool population, was determined using all positive $\mathrm{SAB}$. Positive $\mathrm{SAB}$ were divided into 4 categories: weak (1,000-2,000 MFI), lower moderate (2,001-4,000 MFI), upper moderate (4,001-8,000 MFI) and strong $(>8,000 \mathrm{MFI})$. To determine the titer of these HLA antibodies, positive sera were tested after serial dilutions with phosphate buffered saline (PBS). Reactive beads in the diluted sera were assigned as positive and divided into 
categories as described above.

\section{Luminex single antigen beads C1q testing}

Undiluted positive sera were tested for the presence of complement-fixing antibodies using the modified Luminexbased single antigen beads C1q-assay (C1q Screen - One Lambda Thermo Fisher, West Hills, CA). This assay uses the same single antigen bead set described above but complement-fixing antibodies are detected by a phycoerythrin-conjugated anti-C1q secondary antibody. Sera were tested according to manufacturer instructions and beads with normalized $M F I \geq 500$ were considered positive.

\section{Luminex single antigen beads IgG subclasses testing}

For some cases, positive sera were tested for IgG subclasses using a modified SAB assay, as reported previously (33), replacing the pan-IgG secondary antibody with monoclonal antibodies specific for IgG1, IgG2, IgG3 and IgG4 subclasses (Southern Biotech, Birmingham, AL). Beads with normalized $\mathrm{MFI} \geq 500$ were considered as positive.

\section{Surrogate flow cytometric crossmatch testing}

Sera showing a SAB pattern of weak to lower moderate antibody reactivity, consistent with that seen when binding to multiple beads creating a "MFI dilution effect" (see below), were tested for a flow cytometric crossmatch (FCXM) with mock donor cells to determine the true reactivity strength in-vivo. Briefly, pronase-treated lymphocytes, isolated from whole blood samples of healthy mock donors, were incubated at room temperature with sera containing broadly reacting antibody for 30 minutes. After washing, IgG antibody bound to the mock donor $\mathrm{T}$ and $\mathrm{B}$ cells were detected by addition of a monoclonal anti-human IgG secondary antibody conjugated to a FITC fluorochrome. A positive crossmatch was defined as a median channel shift (MCS) $\geq 150$ from the median channel of the negative control.

\section{HLA epitopes, effect on median-fluorescence intensity (MFI) and testing strategy to determine in-vivo relative strength}

HLA antibodies bind to polymorphic, non-self, amino-acid residues comprising immunogenic epitopes on the surface of the HLA molecule. As shown in Figure 1A, each antigen possesses multiple sites for potential antibody binding. Some epitopes are unique for a specific HLA antigen, while some are shared among several different antigens, and others are present on many HLA antigens.

Patient sera may contain one or more anti-HLA antibodies that can bind to variable numbers of SAB. Reactivity with many SAB may be due to mixture of antibodies directed against multiple HLA epitopes or a single monospecific antibody that recognizes an epitope shared by many HLA antigens. For any specific antibody, the MFI value of each bead is directly affected both by the antibody titer and the number of target epitopes in the $\mathrm{SAB}$ panel. Sera with low to moderate titer, broadly reacting antibodies directed toward an HLA epitope that is shared by a high number of beads may show artificially low MFI values (“diluted MFI value") since a lower number of $\operatorname{IgG}$ molecules are available to bind to each bead. The lower MFI values do not necessarily reflect the antibody titer and $\mathrm{C} 1 \mathrm{q}$ reactivity. Conversely, an antibody with similar specificity, but with high titer, may have a sufficient number of IgG molecules to saturate each bead, resulting in strong MFI values that still persist after serum dilution. At very high titers, these antibodies will also result in a positive $\mathrm{C} 1 \mathrm{q}$ assay. The physiological roles of these antibodies needs to be confirmed by an alternative testing method. FCXM is the test of choice to determine the strength of HLA antibodies in-vivo. This assay is commonly used to assess patient/ donor compatibility and can be performed prospectively or retrospectively. The same assay can also be used to rule in or rule out questionable antibodies, to determine the synergistic effect of multiple weak/low-moderate antibodies and also to determine the in-vivo strength of HLA antibodies that bind to several single antigen beads and have an artificially low MFI value.

Antibodies specific for broadly shared HLA epitopes (e.g., Bw4 or Bw6) are classic examples of this phenomenon, whereby antibody binding can be diluted by the presence of the shared epitope on as many as 25 beads. As shown in Figure 1B, antibody targeting a shared epitope will bind to each bead similarly, but the reduced amount of antibody on each bead will result in lower MFI values. However, when these sera are tested against donor cells in a FCXM, more antibody molecules are available to bind to the smaller number of epitope-sharing HLA antigens present on the lymphocyte cell surface, thus resulting in a positive FCXM. 
A

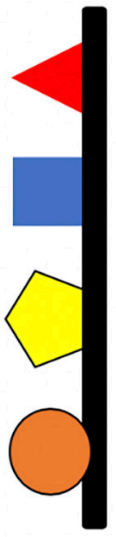

Antigen 1

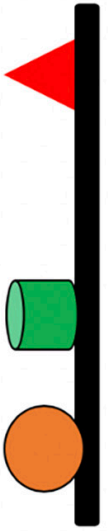

Antigen 2

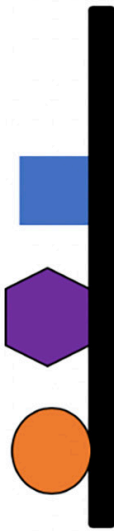

Antigen 3
LEGEND

Epitope shared by antigens 1 and 2

Epitope shared by antigens 1 and 3

$\square$
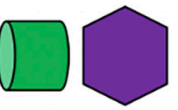

Epitope present only in one antigen

Epitope shared by all antigens
B

\section{1) "DILUTED" MFI VALUE}

- Each SAB assay includes up to 100 different HLA antigens for each Class. Groups of antigens with shared epitopes provide antibodies with many possible target points.

- A low/moderate-titer antibody, targeting an HLA epitope shared by several $(>20)$ single antigen beads may have an artificially low MFI value, not correlating with in-vivo reactivity as observed by FCXM.

\section{2) POSITIVE FLOW CROSSMATCH}

- As compared to single antigen beads, cells express only 6 Class I and up to 8 Class II HLA antigens; hence, fewer shared epitopes are available for antibody binding.

- By virtue of more lgG molecules available, each donor lymphocyte can be bound by a relatively higher amount of IgGs, thus resulting in a positive flow crossmatch.
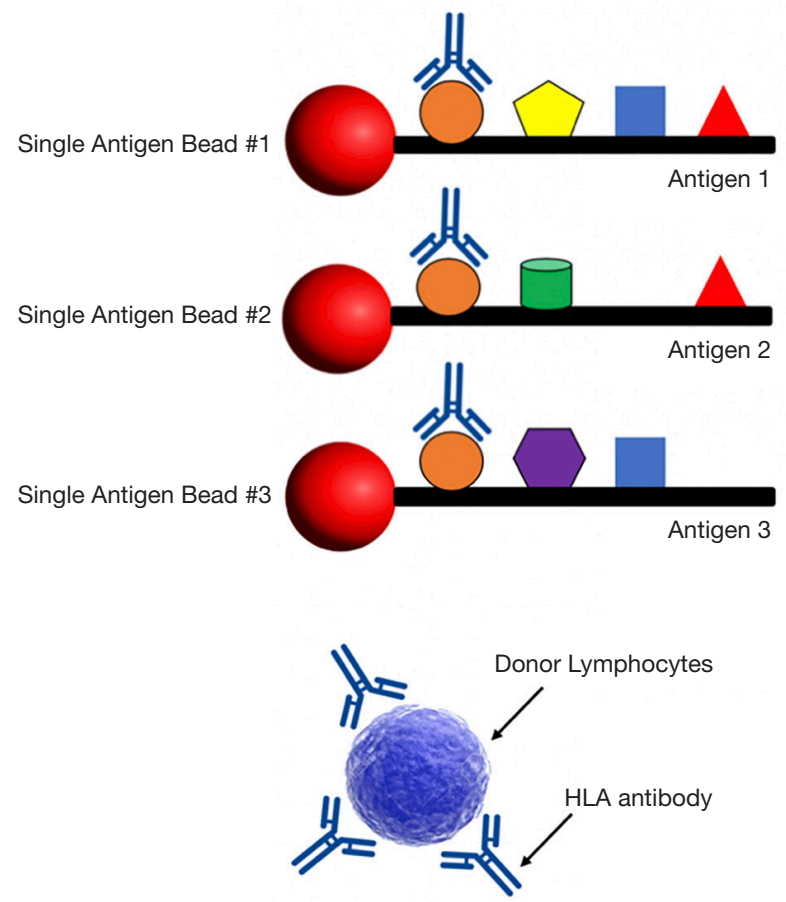

Figure 1 Antibody binding to HLA antigens in Luminex SAB assays and flow cytometry crossmatch. (A) HLA antigens express immunologically relevant epitopes recognized by antibody. Some epitopes are unique for a specific antigen, while others are shared by two or more antigens. The amount of HLA antibody bound to each bead will determine the MFI of each bead. (B) Each SAB is virtually coated with HLA antigens, each of which bear multiple epitopes. An HLA antibody with broad specificity towards an epitope shared by multiple antigens may bind to many different SAB, leading to artificially lower MFI values for each individual SAB as compared to the MFI value seen for an antibody that binds to an epitope on only one or a few SAB. As compared to a panel of SAB, a cell expresses only one or a few antigens that may share an epitope recognized by a broadly reacting antibody. More antibody is available to bind to the shared epitope on the cell surface and can result in a positive flow cytometric crossmatch. SAB, single antigen bead; MFI, median fluorescence intensity. 

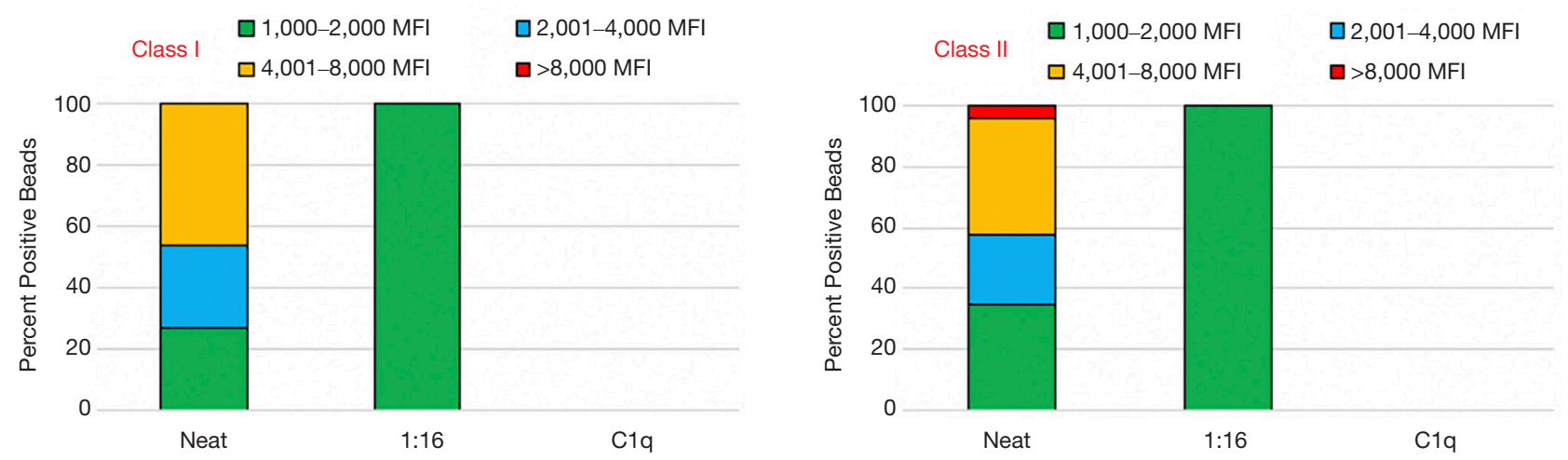

Figure 2 Case \#1: high cPRA/low titer/C1q-negative. Among Class I HLA antibodies, 46\% of positive beads fall in the upper moderate range (4,001-8,000 MFI). The remainder is equally distributed (27\% each) between the weak (1,000-2,000 MFI) and the low moderate (2,001-4,000 MFI) ranges. The Class II show virtually an even distribution of antibodies among the weak, low moderate and upper moderate categories, with only $4 \%$ of antibodies in the strong range (>8,000 MFI). At a 1:16 dilutions, all positive beads fall in the weak range and the C1q assay is negative. MFI, median fluorescence intensity.

\section{Common categories of allosensitized patients}

Among allosensitized patients, there is a great diversity in the spectrum, strength and nature of HLA antibodies. However, one of the most common practices is to riskstratify patients based only on cPRA. In reality, patients with similar cPRA values show a wide range of HLA antibody profiles, with different titers and different complement-fixing properties. Some of the most common HLA antibody profiles are discussed in the examples below.

\section{Representative case \#1: bigh cPRA/low titer/C1q-negative}

A frequent HLA allosensitization profile is that of patients who exhibit a broad profile of HLA Class I and Class II antibodies as determined by $\mathrm{SAB}$ testing. These patients are considered as high cPRA and are deemed difficult to transplant, since identifying a donor with acceptable antigens is not common. However, upon further assessment of the HLA antibody titer and complement binding ability, it becomes evident that, in some cases, these patients can be safely transplanted even in the presence of low titer DSAs.

The example in Figure 2 depicts the profile of the undiluted serum ("neat" column; both Classes) showing the breath of the HLA antibodies for a patient with a cPRA of $90 \%$. Among Class I HLA antibodies, $46 \%$ fall in the upper moderate range $(4,001-8,000 \mathrm{MFI})$. The remaining reactions are equally distributed $(27 \%$ each) between the weak (1,000-2,000 MFI) and the low moderate $(2,001-4,000$ MFI) ranges. The Class II results for the neat serum show virtually an even distribution of antibodies among the weak, low moderate and upper moderate categories, with only $4 \%$ of antibodies in the strong range ( $>8,000 \mathrm{MFI})$. The next step in our algorithm is to determine both the titer and the complement-fixing properties of the HLA antibodies. The dilution study clearly showed that, for both classes, the HLA antibodies expressed by this patient had low titer. As shown in Figure 2 ("1:16" column; both Classes), the diluted antibodies were all in the weak category $(1,000-2,000$ MFI range). The C1q-Luminex assay was negative for both classes and correlated with the low-titer HLA antibody (Figure 2, C1q column; both Classes).

When considering potential donors for patients in this category, the approach is based on the patient clinical status and the risk assessment when choosing between (I) waiting for a donor with acceptable antigens (antibody avoidance), or (II) crossing one or two weak antibodies with early posttransplant monitoring (DSA testing) to determine memory response.

This example clearly illustrates that it is very important to make a distinction between high cPRA profiles, which are based solely on the frequency of unacceptable HLA antigens in the population, from patients with high cPRA and with high titer HLA antibodies. Categorizing patients only by cPRA does not allow for accurate riskstratification, which should be based on the granularity of the HLA antibody profiles and the physiological nature of the antibodies. 

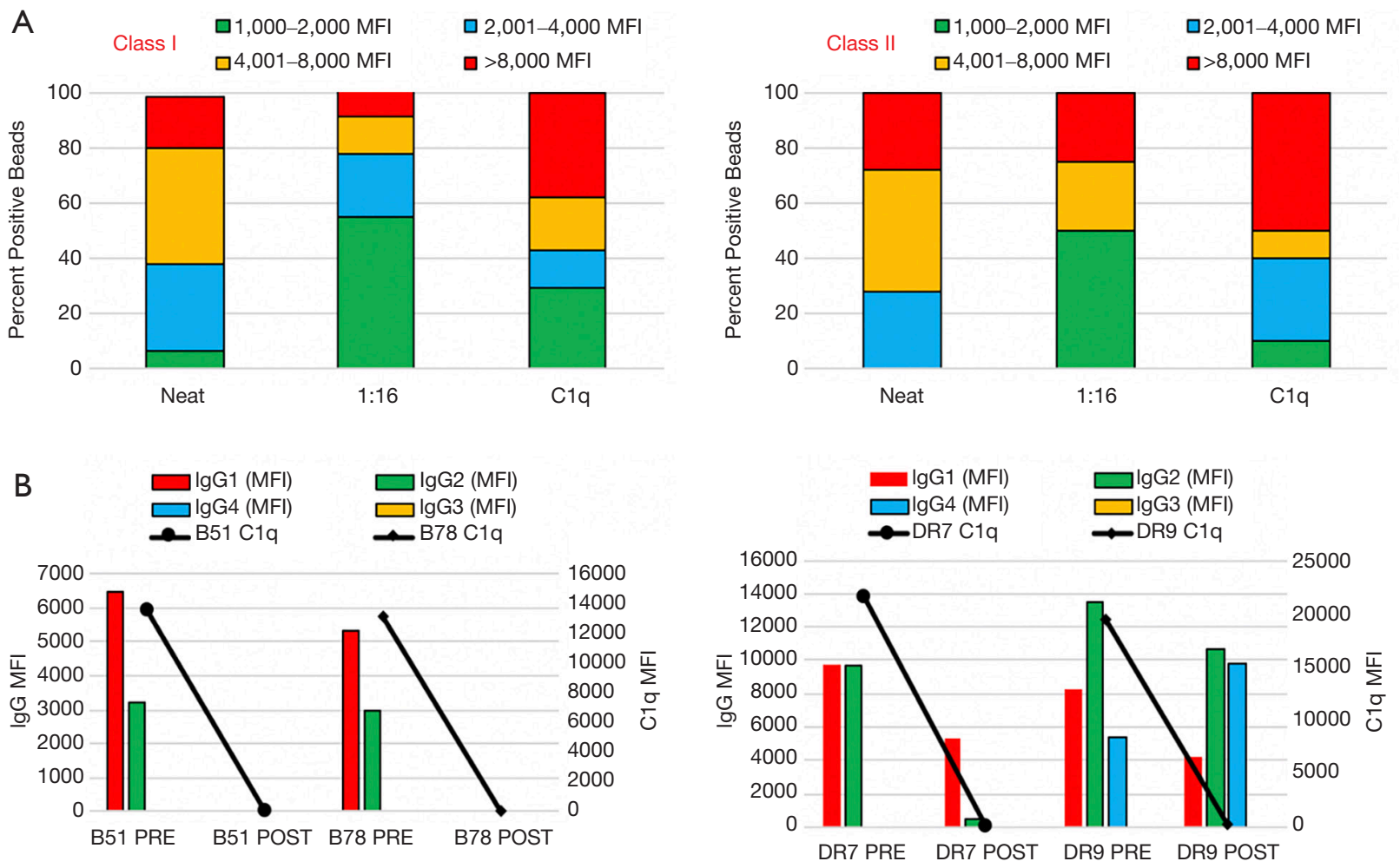

Figure 3 Case \#2: high cPRA/moderate titer/C1q-positive. (A) For Class I, 19\% of antibodies are in the strong (>8,000 MFI) group, 42\% fall in the upper moderate (4,001-8,000 MFI) range, 32\% in the low moderate (2,001-4,000 MFI) range and only $6 \%$ were in the weak range $(1,000-2,000$ MFI). Similarly, on the Class II assay, 28\% were strong (>8,000 MFI), 44\% were in the upper-moderate range (4,001-8,000 MFI), 20\% were in the low moderate range (2,001-4,000 MFI) while none exhibited weak reactivity (1,000-2,000 MFI). For both Classes, 1:16 dilution shows a shift into the weak range with about $55 \%$ of Class I positive beads and $50 \%$ of Class II positive beads in this category. As could be predicted, these antibodies are C1q positive for both Classes and distributed among all ranges. (B) Effect of PLEX and IVIG on IgG subclasses and C1q assay. Despite the relative MFI values of antibodies in the undiluted serum, changes in the subclasses composition of these antibodies following desensitization correlates with the C1q assay results. MFI, median fluorescence intensity; PLEX, plasma exchange; IVIG, intravenous immunoglobulin.

\section{Representative case \#2: bigh cPRA/moderate titer/C1q- positive}

The example in Figure 3 depicts another common HLA allosensitization profile. This is a high cPRA patient expressing moderate titer Class I and Class II HLA antibodies and is C1q-positive.

In the Class I and Class II neat serum, the antibodies are distributed among all four categories. For Class I, 19\% of antibodies are in the strong (>8,000 MFI) group, $42 \%$ fall in the upper-moderate $(4,001-8,000 \mathrm{MFI})$ range, $32 \%$ in the lower moderate $(2,001-4,000 \mathrm{MFI})$ range and only $6 \%$ were in the weak range $(1,000-2,000$ MFI). Similarly, of the Class II HLA antibodies, $28 \%$ were strong $(>8,000$ MFI), $44 \%$ were in the upper moderate range $(4,001-8,000$ MFI), 20\% were in the lower moderate range $(2,001-4,000$
MFI) while none exhibited weak reactivity $(<2,000 \mathrm{MFI})$ (Figure 3A).

To define the nature of these HLA antibodies and their physiological role, we performed dilutions and the C1qbinding assay. For both Classes, the 1:16 dilution clearly showed a shift of reactivity into the weak range $(1,000-2,001$ MFI). In fact, after dilutions, $55 \%$ of Class I antibodies and $50 \%$ of Class II antibodies moved to the weak category (Figure 3A, "1:16" column, both Classes). This indicates that half of the HLA antibodies detected in the undiluted serum can be considered as low titer antibodies. Additionally, the distribution of Class I antibodies in the strong and uppermoderate categories also diminished to $9 \%$ and $14 \%$, respectively. Class II HLA antibodies were instead evenly distributed between the strong and upper-moderate ranges, 


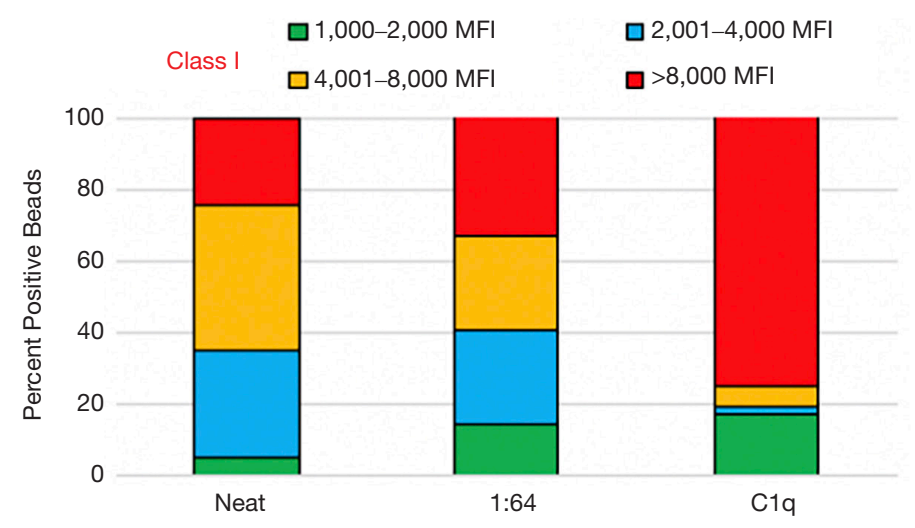

Figure 4 Case \#3: high cPRA/high titer/C1q-positive. The undiluted serum shows an almost even distribution of antibodies among the three groups of MFI ranges between 2001 to $>8,000$. At 1:64 dilution, positive beads remain almost evenly distributed in all ranges. As could be predicted, the $\mathrm{C} 1 \mathrm{q}$ assay is positive and shows that $81 \%$ of the positive beads are in the $>8,000 \mathrm{MFI}$ range. Class II testing was negative. MFI, median fluorescence intensity.

with a noticeable decrease (from $44 \%$ to $35 \%$ ) only in the upper-moderate range. The C1q-Luminex assays reflected this antibody dichotomy and, as could be predicted by the presence of strong and upper moderate antibodies in the 1:16 dilution, it was positive for both Classes (Figure $3 \mathrm{~A}$, C1q column, both Classes).

An interesting aspect of sera with moderate titer antibodies is that therapeutic plasma exchange (PLEX) can often remove enough $\operatorname{IgG}$ antibody to reduce these sera to low titer and C1q-negative reactivity. In fact, when this patient was treated with 4 cycles of PLEX and intravenous immunoglobulin (IVIG), the MFI values and the number of positive beads were significantly decreased; additionally, antibody reactivity for both Classes became C1q-negative (Figure 3B). The number of Class I positive beads decreased $77 \%$ and the MFI range of the remaining positive beads $(\mathrm{n}=7)$ was $1,006-3,180$ MFI (data not shown). For Class II, $76 \%$ of previously positive beads became negative and the serum showed the possible presence of two HLA antibodies, one strong with MFI values $>12,000$ and one with low moderate $\mathrm{MFI}$ values $<3,000$. Interestingly, despite the presence of MFI values $>12,000 \mathrm{MFI}$, the Class II C1q assay was negative. To explain the lack of $\mathrm{C} 1 \mathrm{q}$ reactivity, we evaluated the serum composition for IgG subclasses. As shown in Figure 3B, prior to antibody removal therapy the subclasses composition for the immunodominant antiHLA-B51, -B78 and -DR7 antibodies were mixtures of $\operatorname{IgG1}$ and $\operatorname{IgG} 2$, whereas that of the anti-DR9 antibody was IgG1, IgG2 and IgG4. After PLEX, IgG subclasses were below detection levels for Class I antibodies. For Class II anti-DR7, the sum of MFIs for IgG1 and IgG2 significantly decreased from almost 20,000 to about 5,000 MFI (Figure 3B). In contrast, for DR9 the sum of MFIs for IgG1, IgG2 and IgG4 did not change but the relative ratio of subtypes in the mixture changed. As evident in Figure $3 B$ (right side), the anti-DR9 IgG1 declined while the antiDR9 IgG4 increased. Overall, the subclass composition did not change after treatment. Altogether, these results may suggest that $\mathrm{C} 1 \mathrm{q}$ activation is dependent on the level of HLA antibody and the proportion of each individual $\mathrm{IgG}$ subclass in the mix. Of note, there was no change in Class II IgG subtypes composition when comparing results before and after treatment.

\section{Representative case \#3: high cPRA/high titer/C1q-positive}

Another frequent allosensitization profile is that of patients with high cPRA, high titer and complement-fixing HLA antibodies. The allosensitization profile depicted in Figure 4 is a classic example of such sensitization. The undiluted serum shows an almost even distribution of antibodies among the three groups of MFI ranges between 2,001 to $>8,000$. Interestingly, the 1:64 dilution shows not only that these antibodies have high titer, but also that the number of beads in the $>8,000$ MFI group increases by $10 \%$, probably due to some signal inhibition in the undiluted serum. This reinforces the importance of serum interference removal with EDTA, heat inactivation or DTT. As could be predicted, the C1q assay (Figure 4, last column) is positive and shows that $81 \%$ of the positive beads are in the $>8,000$ MFI range.

This antibody profile represents the most complicated 


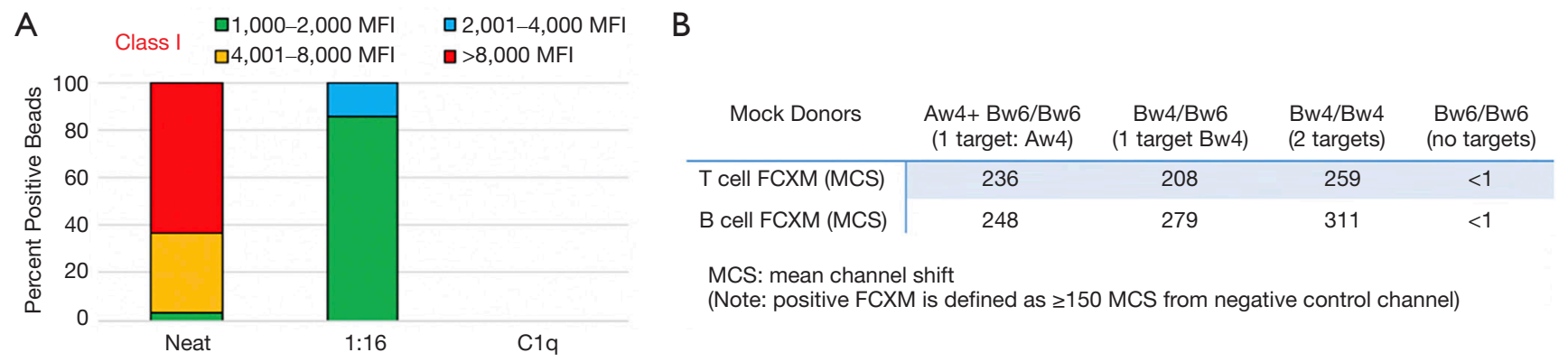

Figure 5 Case \#4: high cPRA/low titer/C1q-negative patients with antibody to a shared HLA epitope. (A) Positive beads distribution for a patient expressing an anti-Bw4 antibody. Despite the fact that $61 \%$ of beads in this serum fall in the $>8,000$ MFI category, the $1: 16$ dilution shows that $86 \%$ of beads have $<2,001 \mathrm{MFI}$ and all are C1q-negative. (B). Cell binding capabilities of the antibodies in this serum. A series of surrogate FCXM against mock donors with one target/cell (Aw4; Bw4/Bw6) or two targets/cell (Bw4/Bw4) were compared to a no target/cell surrogate donor (selflike, Bw6/Bw6 donor). FCXM against one- and two-target donor cells both resulted in a positive FCXM, as indicated by the channel shift observed with all but the self-like mock donor cells. MFI, median fluorescence intensity; FCXM, flow crossmatches.

and difficult to transplant lung allograft cohort. For these patients, the probability of finding an acceptable donor is very low, and desensitization treatments may not be efficient in decreasing or removing HLA antibodies. The small number of antibodies in the weak, low titer range $(<2,001$ MFI) does not allow for increasing the pool of potential donors, even if crossing DSAs is an option. Conversely, the large number of high titer HLA antibodies usually requires several desensitization cycles before observing a decrease, even simply in the MFI values. For these patients, the most frequent choice is antibody avoidance. When appropriate, and based on clinical status, some of these patients can be transplanted crossing weak, low titer antibodies, limiting to crossing no more than two unacceptable antigens, with peri-operative intervention and early DSA monitoring to assess memory response.

\section{Representative case \#4: bigh cPRA/low titer/C1q-negative patients with antibody to a shared eplet}

The serum profile depicted in Figure $5 A$ is the MFI distribution for a patient expressing an anti-Bw4 antibody (cPRA 74\%). In such sera, and despite the MFI value in the undiluted serum, the titer and C1q-fixing capability of an antibody is artificially reduced, due to the "dilution effect" caused by the high numbers of HLA alleles bound to the SABs that can adsorb the antibody. In fact, despite the fact that $61 \%$ of beads in this serum fall in the $>8,000$ MFI category, the $1: 16$ dilution shows that $86 \%$ of beads have $<2,001 \mathrm{MFI}$ and all are C1q-negative. These particular types of sera cannot be necessarily considered as low titer/ C1q-negative because the in-vivo physiology of these antibodies can be very different from what the in-vitro assay may lead us to believe. An antibody like the one shown in Figure $5 \mathrm{~A}$ provides an example to demonstrate the synergistic effect that HLA antibodies have in-vivo. Especially in transplant settings where crossing low titer/ C1q-negative antibodies is an acceptable option, the number of targets in the donor cells will determine the extent of the immunological response against the graft. To demonstrate the synergistic capabilities of the antibodies in this serum, a series of surrogate FCXM were performed. Testing was performed with mock donors with one target per cell $[\mathrm{Bw} 4 / \mathrm{Bw} 6$ and $\mathrm{Aw} 4+\mathrm{Bw} 6 / \mathrm{Bw} 6$ (Aw4 is the Bw4 epitope expressed by a small group of HLA-A antigens)], two targets per cell $(\mathrm{Bw} 4 / \mathrm{Bw} 4)$ and a Bw6 homozygous (self-like) donor. As shown in Figure 5B, FCXM results show that one- and two-target donor cells both result in a significant increase over the background channel shift observed with the Bw6-homozygous target cells. It is also worth mentioning that, the higher the MFI value at the 1:16 dilution, the more likely that the lymphocytotoxic T-cell and B-cell crossmatches (CDC-XM) will be positive, despite the lack of C1q-fixation in the SAB Luminex assay (crossmatch data not shown).

\section{Antibody testing strategy for guiding donor selection in sensitized lung transplant candidate}

Although the examples discussed above are not 


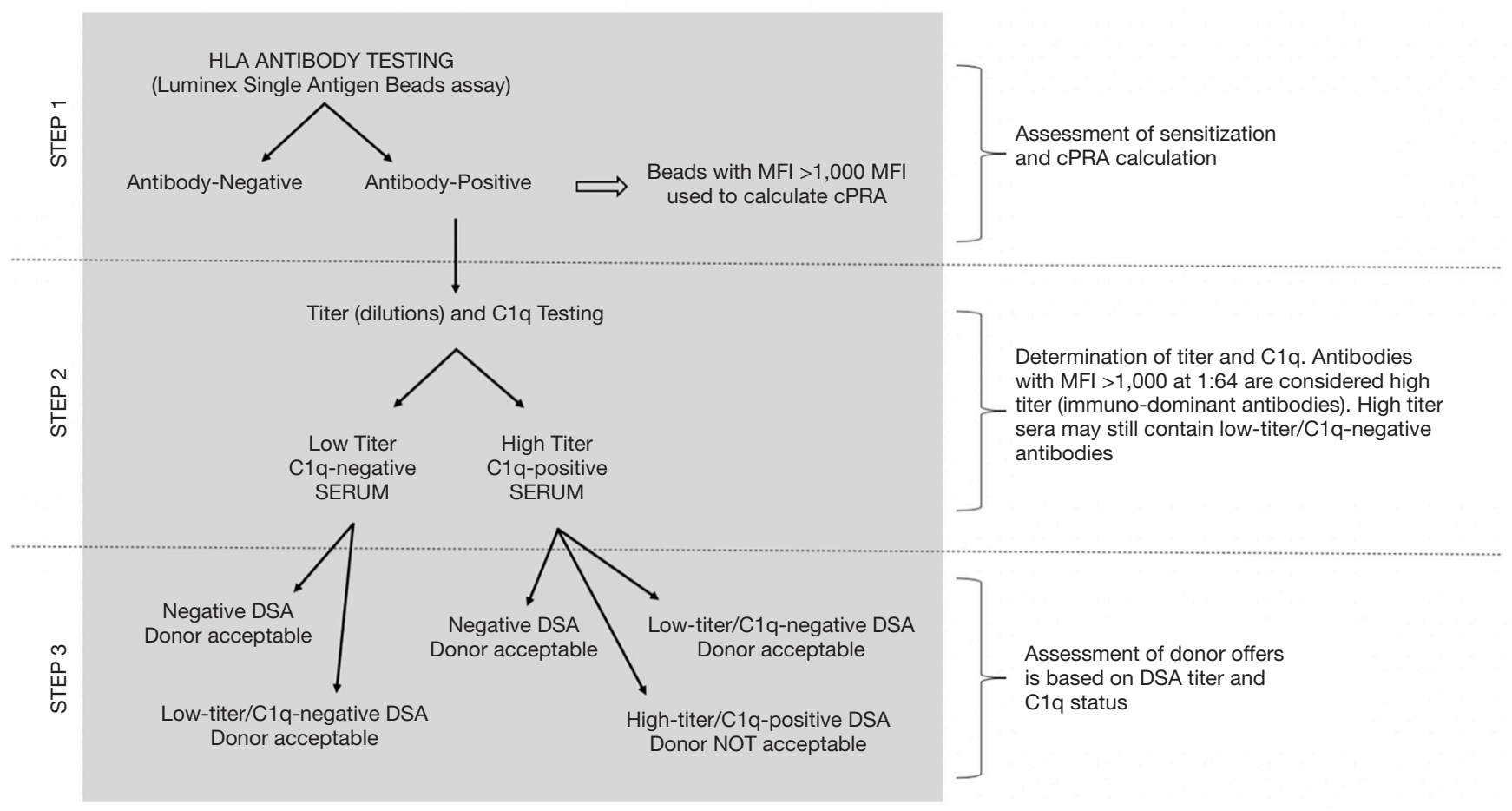

Figure 6 Proposed testing strategy for allosensitized patients. Risk stratification and donor selection is determined by considering the antibody profile in combination with patient clinical status. For each mismatched HLA antigen of a potential donor, the antibody reactivity is evaluated for antibody titer (low-high) and C1q reactivity (negative-positive). MFI, median fluorescence intensity; DSA, donor-specific antibodies.

representative of all allosensitized patients, they do describe the most common profiles and outlines our testing strategy. A common element among these patients is the presence of HLA antibodies. However, identification of the antibody specificity present does not allow, alone, for riskstratification of our candidates.

Here we summarize the steps we take to evaluate the transplantability of a sensitized lung transplant candidate. Figure 6 outlines the steps we take to identify HLA antibodies and determine their physiological significance. First, the presence of HLA antibody is determined by the Luminex SAB assay and we convert the breath of sensitization by entering all the specificities $\geq 1,000 \mathrm{MFI}$ into the UNOS calculator to determine the cPRA. The percent cPRA informs the clinical team whether the patient is highly sensitized (cPRA $>50 \%$ ), indicating a limited donor pool, or if the candidate has a low cPRA $(<50 \%)$ and likely to be offered a donor without the need to cross any potential DSA. Based on the frequency of the acceptable antigens in the donor pool and the clinical status of the candidate, the clinicians may choose to wait for an optimal offer or proceed to transplant by crossing a DSA. Obviously, and regardless of the cPRA value, an offer for a donor that expresses no unacceptable HLA antigens can always be received. Our paper and examples focus on the steps we take to determine the physiological role of HLA antibodies that the clinical team may need to cross due to a limited donor pool and a patient's clinical status.

The next step is to risk-stratify the circulating HLA antibodies, by determining their titer and complement binding ability. These tests are essential to differentiate between HLA antigens that should be avoided from those that are potentially acceptable to cross.

In low cPRA candidates, we anticipate being able to accept donor offers without crossing any DSA. However, based on the clinical status, it may be necessary to consider an offer with positive DSA when the antibody has low titer and is C1q-negative. Additionally, if most of the circulating antibodies are low titer, the patient may avoid any pretransplant desensitization treatment. However, in rare cases, even a low cPRA candidate may require antibody removal therapy when the only option is to transplant in the presence of multiple low-titer DSAs.

In contrast, the chances to identify a donor with negative 
DSA for highly sensitized candidates (cPRA $>50 \%)$, is low, and frequently offers are accepted with one or two low titer, C1q-negative DSAs. However, if the majority of HLA antibodies are high titer and C1q-binding, desensitization should be considered as an option to improve the chances to find a suitable donor. The efficacy of desensitization will be assessed based on the percent of antibodies that drop to low titer $(<1: 16)$ post-treatment. In most cases, the circulating antibodies consist of a mixture of HLA antibodies with different titers and specificities that will impact the response to therapy.

\section{Discussion}

Preformed donor-specific HLA antibodies are recognized as a barrier for lung transplantation and may limit a sensitized candidate to find a suitable donor $(1,2)$. Our approach outlined in the examples discussed above provides an individualized strategy to evaluate each candidate, not only for the specificity of circulating HLA antibody, but also for the physiological nature of the HLA antibody by determining titer and complement-fixing properties. This detailed antibody characterization, along with the patient's clinical status, represents the foundation of risk stratification and can improve not only access to donors, but also graft outcome.

Furthermore, not all sensitized candidates need to be subjected to antibody removal protocols. For some patients, a detailed determination of the HLA antibody, along with the prediction of the pool of potential donors after avoiding all DSA or after accepting low titer DSAs, may improve the odds of finding an acceptable donor. In contrast, for patients with high cPRA and high-risk HLA antibodies, the options are limited and the probability of finding a suitable donor are lower. Those candidates should be considered for desensitization and the efficacy of treatment should be closely monitored.

A US Food and Drug Administration (FDA) workshop, focused on determining which assays should be used for antibody quantitation, concluded that the Luminex SAB assay alone cannot be used as a guidance for evaluating desensitization strategies (34). Tambur and colleagues reported that dilution studies can overcome many of the limitations of the $\mathrm{SAB}$ assay that are frequently observed in the presence of undiluted patient serum, such as prozone effect (inhibition) and beads saturation (25). In contrast to neat SAB MFI level, titration of HLA antibody may provide a more accurate metric of the antibody strength (25).
Similarly, titration studies are essential to determine the efficacy of desensitization. A study conducted in three centers involving 38 patients that received several different protocols of antibody removal therapy, demonstrated that antibody titration can serve as a tool to measure the efficacy of pre-transplant desensitization protocols $(25,35)$.

Protocol perioperative treatment of lung transplant patients with a cPRA $>30 \%$ and with pre-formed DSA or PRA-positive/DSA-negative, showed similar transplant outcomes as compared to unsensitized candidates, suggesting that lung transplantation can be safely performed in sensitized patients (17). The main limitation of the study was the lack of information on the level of DSA crossed, as only neat MFI values were recorded pre-treatment and compared to post-treatment testing results. Only 5/53 patients in the DSA-positive cohort had a positive CDC-XM, suggesting that in most cases the threshold of pre-formed DSA was low risk. Furthermore, in some patients the DSA was present only in historical sera and the maximum cPRA in the cohort was only $62 \%$. Overall, $53 \%$ of treated patients had one persistent DSA posttransplant and the pre-formed DSA MFI was not predictive of the response to therapy. In contrast, in a cohort of lung transplant recipients treated for AMR with a Carfilzomib regiment, we showed that the efficacy of treatment correlates with the DSA titer and not MFI (36). Similarly, Pinelli et al. showed that antibodies with pre-treatment titer up to $1: 512$ could be effectively lowered to a transplantable level, while those with titer above 1:512 could not be reliably removed using PLEX and IVIG (35).

In our risk-stratification strategy, we also consider the ability of DSA to bind C1q, since this functional characteristic is essential to predict which DSA poses the higher risk for rejection. Complement binding DSA has been associated with DSAs with a titer $>1: 16$ (27), and with the mixture of $\operatorname{IgG}$ subtypes that can most effectively activate the complement cascade, including IgG1 and IgG3 $(25,33)$. In cases when crossing a DSA is the only option to provide the lung candidate a life-saving procedure, this can be safely done only in the presence of low-titer, C1q-negative DSAs. It is worth mentioning that, to avoid complications caused by an uptake in DSA titer due to memory responses, these patients need to be closely monitored in the immediate aftermath of transplant and frequently in the initial months after.

In summary, the main goal of the histocompatibility clinical laboratory is to use all the tools available to facilitate safe access of sensitized lung transplant candidates to 
potential donors. The ultimate decision is based on the clinical team's assessment of several parameters, leading to a risk-stratification that is unique for each patient. To guide pre- and post-transplant decisions, close interaction between the HLA laboratory and the clinical team is essential to interpret results of the various assays and frame the physiological nature of the HLA antibodies.

\section{Acknowledgments}

Funding: None.

\section{Footnote}

Provenance and Peer Review: This article was commissioned by the Guest Editor (Jonathan D'Cunha) for the series "Lung Transplantation: Past, Present, and Future" published in Fournal of Thoracic Disease. The article has undergone external peer review.

Conflicts of Interest: All authors have completed the ICMJE uniform disclosure form (available at: https:// dx.doi.org/10.21037/jtd-2021-21). The series "Lung Transplantation: Past, Present, and Future" was commissioned by the editorial office without any funding or sponsorship. All authors have no other conflicts of interest to declare.

Ethical Statement: All authors are accountable for all aspects of the work in ensuring that questions related to the accuracy or integrity of any part of the work are appropriately investigated and resolved.

Open Access Statement: This is an Open Access article distributed in accordance with the Creative Commons Attribution-NonCommercial-NoDerivs 4.0 International License (CC BY-NC-ND 4.0), which permits the noncommercial replication and distribution of the article with the strict proviso that no changes or edits are made and the original work is properly cited (including links to both the formal publication through the relevant DOI and the license). See: https://creativecommons.org/licenses/by-nc-nd/4.0/.

\section{References}

1. Tague LK, Witt CA, Byers DE, et al. Association between Allosensitization and Waiting List Outcomes among Adult Lung Transplant Candidates in the United States. Ann Am Thorac Soc 2019;16:846-52.
2. Aversa M, Benvenuto L, Kim H, et al. Effect of Calculated Panel Reactive Antibody Value on Waitlist Outcomes for Lung Transplant Candidates. Ann Transplant 2019;24:383-92.

3. Tikkanen JM, Singer LG, Kim SJ, et al. De Novo DQ Donor-Specific Antibodies Are Associated with Chronic Lung Allograft Dysfunction after Lung Transplantation. Am J Respir Crit Care Med 2016;194:596-606.

4. Reinsmoen NL, Lai CH, Mirocha J, et al. Increased negative impact of donor HLA-specific together with nonHLA-specific antibodies on graft outcome. Transplantation 2014;97:595-601.

5. Hachem RR, Yusen RD, Meyers BF, et al. Anti-human leukocyte antigen antibodies and preemptive antibodydirected therapy after lung transplantation. J Heart Lung Transplant 2010;29:973-80.

6. Brugière $\mathrm{O}$, Suberbielle $\mathrm{C}$, Thabut $\mathrm{G}$, et al. Lung transplantation in patients with pretransplantation donor-specific antibodies detected by Luminex assay. Transplantation 2013;95:761-5.

7. Snyder LD, Gray AL, Reynolds JM, et al. Antibody desensitization therapy in highly sensitized lung transplant candidates. Am J Transplant 2014;14:849-56.

8. Appel JZ, 3rd, Hartwig MG, Davis RD, et al. Utility of peritransplant and rescue intravenous immunoglobulin and extracorporeal immunoadsorption in lung transplant recipients sensitized to HLA antigens. Hum Immunol 2005;66:378-86.

9. Feingold B, Bowman P, Zeevi A, et al. Survival in allosensitized children after listing for cardiac transplantation. J Heart Lung Transplant 2007;26:565-71.

10. Mahle WT, Tresler MA, Edens RE, et al. Allosensitization and outcomes in pediatric heart transplantation. J Heart Lung Transplant 2011;30:1221-7.

11. Kransdorf EP, Kittleson MM, Patel JK, et al. Calculated panel-reactive antibody predicts outcomes on the heart transplant waiting list. J Heart Lung Transplant 2017;36:787-96.

12. Park JE, Kim CY, Park MS, et al. Prevalence of pretransplant anti-HLA antibodies and their impact on outcomes in lung transplant recipients. BMC Pulm Med 2018;18:45.

13. Hadjiliadis D, Chaparro C, Reinsmoen NL, et al. Pretransplant panel reactive antibody in lung transplant recipients is associated with significantly worse posttransplant survival in a multicenter study. J Heart Lung Transplant 2005;24:S249-54.

14. Bosanquet JP, Witt CA, Bemiss BC, et al. The impact of 
pre-transplant allosensitization on outcomes after lung transplantation. J Heart Lung Transplant 2015;34:1415-22.

15. Safavi S, Robinson DR, Soresi S, et al. De novo donor HLA-specific antibodies predict development of bronchiolitis obliterans syndrome after lung transplantation. J Heart Lung Transplant 2014;33:1273-81.

16. Kobashigawa J, Colvin M, Potena L, et al. The management of antibodies in heart transplantation: An ISHLT consensus document. J Heart Lung Transplant 2018;37:537-47.

17. Tinckam KJ, Keshavjee S, Chaparro C, et al. Survival in sensitized lung transplant recipients with perioperative desensitization. Am J Transplant 2015;15:417-26.

18. Lefaucheur C, Loupy A. Antibody-Mediated Rejection of Solid-Organ Allografts. N Engl J Med 2018;379:2580-2.

19. Butler CL, Valenzuela NM, Thomas KA, et al. Not All Antibodies Are Created Equal: Factors That Influence Antibody Mediated Rejection. J Immunol Res 2017;2017:7903471.

20. Valenzuela NM, Reed EF. Antibody-mediated rejection across solid organ transplants: manifestations, mechanisms, and therapies. J Clin Invest 2017;127:2492-504.

21. Tait BD, Susal C, Gebel HM, et al. Consensus guidelines on the testing and clinical management issues associated with HLA and non-HLA antibodies in transplantation. Transplantation 2013;95:19-47.

22. Kiernan JJ, Ellison CA, Tinckam KJ. Measuring alloantibodies: a matter of quantity and quality. Curr Opin Organ Transplant 2019;24:20-30.

23. Lee H, Min JW, Kim JI, et al. Clinical Significance of HLA-DQ Antibodies in the Development of Chronic Antibody-Mediated Rejection and Allograft Failure in Kidney Transplant Recipients. Medicine (Baltimore) 2016;95:e3094.

24. Wozniak LJ, Hickey MJ, Venick RS, et al. Donor-specific HLA Antibodies Are Associated With Late Allograft Dysfunction After Pediatric Liver Transplantation. Transplantation 2015;99:1416-22.

25. Tambur AR, Herrera ND, Haarberg KM, et al. Assessing Antibody Strength: Comparison of MFI, C1q, and Titer Information. Am J Transplant 2015;15:2421-30.

26. Wiebe C, Nickerson P. Posttransplant monitoring of de novo human leukocyte antigen donor-specific antibodies in kidney transplantation. Curr Opin Organ Transplant
2013;18:470-7.

27. Zeevi A, Lunz J, Feingold B, et al. Persistent strong anti-HLA antibody at high titer is complement binding and associated with increased risk of antibody-mediated rejection in heart transplant recipients. J Heart Lung Transplant 2013;32:98-105.

28. Loupy A, Lefaucheur C, Vernerey D, et al. Complementbinding anti-HLA antibodies and kidney-allograft survival. N Engl J Med 2013;369:1215-26.

29. Bouquegneau A, Loheac C, Aubert O, et al. Complementactivating donor-specific anti-HLA antibodies and solid organ transplant survival: A systematic review and metaanalysis. PLoS Med 2018;15:e1002572.

30. Kim JJ, Shaw O, Martin C, et al. Clinical risk stratification of paediatric renal transplant recipients using $\mathrm{C} 1 \mathrm{q}$ and C3d fixing of de novo donor-specific antibodies. Pediatr Nephrol 2018;33:167-74.

31. Lan JH, Gjertson D, Zheng Y, et al. Clinical utility of complement-dependent C3d assay in kidney recipients presenting with late allograft dysfunction. Am J Transplant 2018;18:2934-44.

32. Couchonnal E, Rivet C, Ducreux S, et al. Deleterious impact of C3d-binding donor-specific anti-HLA antibodies after pediatric liver transplantation. Transpl Immunol 2017;45:8-14.

33. Lefaucheur C, Viglietti D, Bentlejewski C, et al. IgG Donor-Specific Anti-Human HLA Antibody Subclasses and Kidney Allograft Antibody-Mediated Injury. J Am Soc Nephrol 2016;27:293-304.

34. Archdeacon P, Chan M, Neuland C, et al. Summary of FDA antibody-mediated rejection workshop. Am J Transplant 2011;11:896-906.

35. Pinelli DF, Zachary AA, Friedewald JJ, et al. Prognostic tools to assess candidacy for and efficacy of antibodyremoval therapy. Am J Transplant 2019;19:381-90.

36. Ensor CR, Yousem SA, Marrari M, et al. Proteasome Inhibitor Carfilzomib-Based Therapy for AntibodyMediated Rejection of the Pulmonary Allograft: Use and Short-Term Findings. Am J Transplant 2017;17:1380-8.

Cite this article as: Mangiola $M$, Marrari $M, X u$ Q, Sanchez PG, Zeevi A. Approaching the sensitized lung patient: risk ssessment for donor acceptance. J Thorac Dis 2021;13(11):67256736. doi: $10.21037 /$ jtd-2021-21 